\title{
WHEN IS A FLAT ALGEBRA OF FINITE TYPE?
}

\author{
PETER SCHENZEL
}

(Communicated by Louis J. Ratliff, Jr.)

\begin{abstract}
Let $A$ denote a commutative Noetherian domain. For an intermediate ring $A \subseteq B \subseteq A_{x}$ flat over $A$, it is shown that $B$ is an $A$-algebra of finite type. This is followed by an intrinsic description of the flatness of $B$ over $A$ and the asymptotic behavior of certain prime divisors. As an application, flat ideal-transforms are characterized.
\end{abstract}

Let $A$ denote a commutative Noetherian domain. Let $\mathbb{Q}(A)$ be the quotient field of $A$. By an intermediate ring we understand a commutative ring $B$ with $A \subseteq B \subseteq \mathbb{Q}(A)$. In his paper [R], Richman proved that an intermediate ring $B$ is a Noetherian ring provided $B$ is flat over $A$. In general $B$ is not an $A$-algebra of finite type. For instance, take $A$ the integers and $B$ the rationals. If $B$ is an intermediate ring of finite type over $A$, then $B \subseteq A_{x}$ for a certain $0 \neq x \in A$. This necessary condition is also sufficient for an $A$-flat intermediate ring $B$ being of finite type over $A$ as shown in the following

Theorem 1. Let $B$ be an intermediate ring with $A \subseteq B \subseteq A_{x}, x \neq 0$. Suppose that $B$ is flat as an A-module. Then $B$ is an A-algebra of finite type.

Here $A_{x}$ denotes the localization of $A$ with respect to $\left\{x^{n}: n \in \mathbb{N}\right\}$. To prove Theorem 1 we need a few preliminaries about certain asymptotic prime divisors and flatness.

Proposition. Let $B$ be an intermediate ring with $A \subseteq B \subseteq A_{x}$. Then

$$
\operatorname{Ass}_{A}\left(x^{n} B \cap A / x^{n} A\right), n \geq 1,
$$

forms an increasing set of prime ideals stabilizing for large $n$ to a finite set equal to $\operatorname{Ass}_{A}(B / A)$.

Proof. First we will show that the considered sets of associated prime ideals are increasing. This follows easily by virtue of the monomorphism

$$
x^{n} B \cap A / x^{n} A \rightarrow x^{n+1} B \cap A / x^{n+1} A, \quad n \geq 1,
$$

Received by the editors March 23, 1989.

1980 Mathematics Subject Classification (1985 Revision). Primary 13C11, 13E05; Secondary $14 \mathrm{~A} 15,14 \mathrm{~B} 25$.

Key words and phrases. Intermediate ring, flat algebra, algebra of finite type, prime divisor, ideal-transform, affine scheme. 
defined by $r+x^{n} A \mapsto r x+x^{n+1} A$. Because of

$$
\operatorname{Ass}_{A}\left(x^{n} B \cap A / x^{n} A\right) \subseteq \operatorname{Ass}_{A} A / x^{n} A=\operatorname{Ass}_{A} A / x A,
$$

note that $x$ is a nonzero divisor, the desired sets stabilize for large $n$ to a finite set.

Now let $P \in \operatorname{Ass}_{A}\left(x^{n} B \cap A / x^{n} A\right)$. Then there exists an element $r \in x^{n} B \cap$ $A \backslash x^{n} A$ such that $P=x^{n} A: r$. Put $q=r / x^{n} \in B$. It follows that $q \notin A$ and $P=A: q$, as can be easily seen. That is, $P \in \operatorname{Ass}_{A}(B / A)$. In order to prove the reverse inclusion let $P \in \operatorname{Ass}_{A}(B / A)$. Then there is an element $q=r / x^{n} \in B \backslash A, r \in A$, such that $P=A: q$. As above, $P=x^{n} A: r$ and $r \in x^{n} B \cap A \backslash x^{n} A$. That is, $P \in \operatorname{Ass}_{A}\left(x^{n} B \cap A / x^{n} A\right)$, now, by the previous consideration the claim follows.

As an additional feature to our investigations we will characterize when an intermediate ring $A \subseteq B \subseteq A_{x}$ is flat over $A$. This is done in

Theorem 2. An intermediate ring $B$ with $A \subseteq B \subseteq A_{x}, x \neq 0$, is flat as an $A$-module if and only if $B=P B$ for all $P \in \operatorname{Ass}_{A}(B / A)$.

Proof. Suppose $B$ is flat as an $A$-module. Let $P B$ be a proper ideal for some $P \in \operatorname{Ass}_{A}(B / A)$. By the Going Down theorem, see [M], there exists a prime ideal $Q$ minimal over $P B$ such that $P=Q \cap A$. The induced homomorphism $A_{P} \rightarrow B_{Q}$ makes $B_{Q}$ into a faithful flat $A_{P}$-module. Because of $B_{Q} \subseteq \mathbb{Q}\left(A_{P}\right)$ it yields $B_{Q}=A_{P}$ and $A_{P}=B_{P}$, contracting the choice of $P$ as an element of $\operatorname{Ass}_{A}(B / A)$. Now suppose that $B=P B$ for all prime ideals $P \in \operatorname{Ass}_{A}(B / A)$. To show that $B$ is flat over $A$, it is enough to prove that $B_{Q}$ is flat over $A_{P}$ for all $Q \in \operatorname{Spec} B$ and $P=Q \cap A$, see [M, (3.J)]. Let $Q \in \operatorname{Spec} B$. Then $P=Q \cap A \notin \operatorname{Ass}_{A}(B / A)$ because $Q \supseteq P B$ is a proper ideal. If $P \notin \operatorname{Supp}_{A}(B / A)$, then $A_{P}=B_{P}=B_{Q}$ and $B_{Q}$ is flat over $A_{P}$. Suppose $P \in \operatorname{Supp}_{A}(B / A)$. Then there is a prime ideal $P^{\prime} \in \operatorname{Ass}_{A}(B / A)$ such that $P^{\prime} \subseteq P$. Note that $\operatorname{Supp}_{A}(B / A)$ and $\operatorname{Ass}_{A}(B / A)$ have the same set of minimal prime ideals, see $[\mathrm{B}, \S 1,3$, Corollary 1$]$. But then

$$
B=P^{\prime} B \subseteq P B \subseteq Q \subseteq B,
$$

contracting the choice of $Q$.

With the previous notations, set

$$
I=x^{n} A:\left(x^{n} B \cap A\right),
$$

where $n$ is chosen such that $\operatorname{Ass}_{A}\left(x^{n} B \cap A / x^{n} A\right)$ stabilizes. Note that $I=$ $\mathrm{Ann}_{A}\left(x^{n} B \cap A / x^{n} A\right)$.

Corollary 1. An intermediate ring $B$ as above is flat as an $A$-module if and only if $B=I B$.

Proof. Because $\operatorname{Ass}_{A}(B / A)=\operatorname{Ass}_{A}\left(x^{n} B \cap A / x^{n} A\right)$ it follows, see [B, $\S 1,4$, Theorem 2], that $I$ and $\operatorname{Ass}_{A}(B / A)$ have the same set of minimal prime ideals. Then the claim follows by the theorem. 
For an ideal $I$ of $A$ let

$$
T(I)=\left\{q \in \mathbb{Q}(A): I^{n} q \subseteq A \text { for some } n \in \mathbb{N}\right\}
$$

denote the ideal-transform of $A$ with respect to $I$. Note that $T(I)$ is an intermediate ring with $A \subseteq T(I) \subseteq A_{x}, 0 \neq x \in I$.

Corollary 2. The ideal-transform $T(I)$ is flat over $A$ if and only if $T(I)=$ $J T(I)$, where $J$ denotes the intersection of those primary components of a reduced primary decomposition of $x A, 0 \neq x \in I$, for which the associated prime ideals contain $I$.

Proof. First note that

$$
x T(I) \cap A=x A:\langle I\rangle=\bigcup_{k \geq 1} x A: I^{k} .
$$

Furthermore $\operatorname{Ass}_{A}\left(x^{n} T(I) \cap A / x^{n} A\right)=$ Ass $A / x A \cap V(I)$ for all $n \geq 1$. As can be easily seen, $J=x A:(x A:\langle I\rangle)$. Because $J$ and $\operatorname{Ann}_{A}\left(x^{n} T(I) \cap A / x^{n} A\right)$ have the same radical, Corollary 1 proves the claim.

The previous corollary generalizes part of [E, (3.2)]. For a further investigation about the flatness of ideal-transforms see also [S].

Corollary 3. With the notations of Corollary 1 , an intermediate ring $B$ is flat over $A$ if and only if $T(I)=B$ and $\operatorname{Spec} A \backslash V(I)$ is an affine scheme.

Proof. It holds in general that if $I$ is an ideal of a Noetherian domain $A$, then the quasi-affine scheme $\operatorname{Spec} A \backslash V(I)$ is affine if and only if $T(I)=I T(I)$, see $[\mathrm{H}]$. By Corollary 1 this proves the "only if" part of the statement. Assume that $B$ is flat over $A$, i.e., $B=I B$ by Corollary 1 . It is enough to prove that $T(I)=B$. First note that $T(I) \subseteq T(I B)=B$. On the other side let $r / x^{n} \in B$. Then $r \in x^{n} B \cap A$. Because

$$
\operatorname{Supp}_{A}\left(x^{n} B \cap A / x^{n} A\right) \subseteq V(I)
$$

there is an integer $m$ that $I^{m} r \subseteq x^{n} A$, i.e., $r / x^{n} \in T(I)$ as required.

In particular, let $J$ be an ideal of a Noetherian domain $A$ such that Spec $A \backslash V(J)$ is an affine scheme. Then $T(J)$ is flat over $A$ and an $A$-algebra of finite type by Theorem 1.

Proof of Theorem 1. Let $I=x^{n} A:\left(x^{n} B \cap A\right)$ as defined above. By [B], $\operatorname{Ass}_{A}(B / A)$ and $\operatorname{Supp}_{A}(B / A)$ have the same set of minimal prime ideals containing $I$. Therefore $\operatorname{Supp}_{A}(B / A) \subseteq V(I)$ because of $I=\operatorname{Ann}_{A}\left(x^{n} B \cap A / x^{n} A\right)$ and the proposition. By Corollary $1, B=I B$, i.e., there is a decomposition of the unit

$$
1=\sum_{i=1}^{s} r_{i} q_{i}, r_{i} \in I, \quad q_{i} \in B, \quad i=1, \ldots, s .
$$

Now we claim that $B=A\left[q_{1}, \ldots, q_{s}\right]$. Clearly $B \supseteq A\left[q_{1}, \ldots, q_{s}\right]$. Let $y \in B$. Because of $\operatorname{Supp}_{A}(B / A) \subseteq V(I)$ there is an integer $n$ such that $I^{n} y \subseteq A$. The 
$n$th power of the above relation yields

$$
1=\sum_{|a|=n} r_{a} q^{a}, \quad a=\left(a_{1}, \ldots, a_{s}\right)
$$

with $r_{a} \in I^{n}$. Therefore

$$
y=\sum_{|a|=n}\left(r_{a} y\right) q^{a} \in A\left[q_{1}, \ldots, q_{s}\right],
$$

as required.

\section{REFERENCES}

[B] N. Bourbaki, Algèbre commutative, Hermann, Paris, 1961, Chap. 4.

[E] P. M. Eakin, Jr., W. Heinzer, D. Katz, and L. J. Ratliff, Jr., Note on ideal-transforms, Rees rings, and Krull rings, J. Algebra 110 (1987), 407-419.

[H] R. Hartshorne, Algebraic geometry, Springer-Verlag, New York, Heidelberg, and Berlin, 1977.

[M] H. Matsumura, Commutative algebra, 2nd ed., Benjamin, New York, 1980.

[R] F. Richman, Generalized quotient rings, Proc. Amer. Math. Soc. 16 (1965), 794-799.

[S] P. Schenzel, Flatness and ideal-transforms of finite type, (to appear).

Sektion Mathematik der Martin-Luther-Universität Halle-Wittenberg, Postfach, Halle, DDR-4010, German Democratic Republic 